\title{
GABBR1 has a HERV-W LTR in its regulatory region - a possible implication for schizophrenia
}

Hedi Hegyi $i^{1,2}$

\begin{abstract}
Schizophrenia is a complex disease with uncertain aetiology. We suggest GABBR1, GABA receptor B1 implicated in schizophrenia based on a HERV-W LTR in the regulatory region of GABBR1. Our hypothesis is supported by: (i) GABBR1 is in the 6p22 genomic region most often implicated in schizophrenia; (ii) microarray studies found that only presynaptic pathway-related genes, including GABA receptors, have altered expression in schizophrenic patients and (iii) it explains how HERV-W elements, expressed in schizophrenia, play a role in the disease: by altering the expression of GABBR1 via a long terminal repeat that is also a regulatory element to GABBR1.
\end{abstract}

Reviewers: This paper was reviewed by Sandor Pongor and Martijn Huynen.

Keywords: Schizophrenia, Human endogenous retrovirus, HERV-W, long terminal repeat, LTR, GABA, GABBR1, GABA receptor, Enhancer, Silencer

\section{Background}

Schizophrenia is a devastating psychiatric disorder with a strong genetic component (with an estimated $80-85 \%$ heritability [1]) affecting approximately $1 \%$ of the population. Despite intensive research in the past decades there is still no known cause of the disease that would account for the majority of the cases. Several systematic studies have been conducted that implicated various genes in the aetiology of schizophrenia (in Genecards [2], a comprehensive knowledgebase of all human genes, more than 1600 genes are annotated to have some association with schizophrenia), however only a few of them are considered susceptibility genes [1,3]. In a meta-analysis of more than 8000 European-ancestry subjects significant association with schizophrenia was observed in a region of linkage disequilibrium on chromosome 6p22.1, a region that has been commonly implicated in schizophrenia for a long time [4].

A comprehensive microarray expression profiling of the prefrontal cortex pinpointed a group of genes with abnormal expression [5]. Mirnics et al. found that out of 250 gene groups only genes in the presynaptic pathway showed an altered expression [5]. This is in line with the findings of other groups, calling schizophrenia the disease of the synapse [6].

\footnotetext{
Correspondence: hegyihedi@gmail.com

${ }^{1}$ CEITEC - Central European Institute of Technology, Masaryk University, CZ-62500, Brno, Czech Republic

${ }^{2}$ Institute of Enzymology, Research Centre for Natural Sciences, Hungarian Academy of Sciences, Budapest, Hungary
}

In a different approach, mostly subscribed to the hypothesis of viral origin of schizophrenia, several groups found a marked expression of human endogenous retroviruses, mostly the HERV-W subtype being expressed in patients with recent onset of schizophrenia [7,8]. Endogenous retroviruses, both HERV-W and HERV-H, were also reported in patients of multiple sclerosis and other types of autoimmune diseases $[9,10]$.

Several studies implied the GABA receptors in the aetiology of schizophrenia. Besides the study by Mirnics et al. [5] other groups also found the GABA receptor B1 gene (GABBR1) to be downregulated in schizophrenic patients $[3,11,12]$.

Several papers implicated the $6 \mathrm{p} 22$ chromosomal region in schizophrenia, based on genetic studies of familial cases and large cohorts $[4,13]$. A review outlined a long genomic region to be associated with the disease, encompassing $25 \mathrm{Mb}$ of the $6 \mathrm{p}$ genomic region in question [14]. However, several of the common variants implicated in schizophrenia showing a significant association were not associated with any particular genes [4]. This also leads us to the question of genetic changes that are outside of the coding region of protein-coding genes. This is becoming ever more evident as less single nucleotide variants are found to be associated with genetic diseases that affect the protein sequence than found in regulatory regions [15].

However, only sporadic knowledge existed till now regarding the regulatory regions of genes that might 
affect the expression of genes implicated in genetic diseases. A recent publication has identified $\sim 2.9$ million DNase I hypersensitive sites (DHSs), markers of regulatory DNA in the human genome, based on the coexpression patterns of the regions and known promoters of all human protein-coding genes [16]. The authors did not investigate if these regulatory elements are enhancers, silencers, insulators or other types of regulators.

\section{Findings}

Here we analyzed these regulatory regions of the human genome for the presence of the long terminal region (LTR) of the human endogenous virus type W (HERV-W) as this type of HERV was identified in patients with recent-onset schizophrenia [7]. We used the LTR as query as opposed to the full sequence of HERV-W because it is known to have enhancer/promoter/regulatory functions [17].

Using the HERV-W long terminal repeat (LTR) as query against the regulatory sequences identified in the study of Thurman et al. [16] revealed a full-length match in the regulatory region of GABBR1, the GABA receptor B1 gene (Table 1). GABBR1 is located in the 6p22 chromosomal region that has been implicated in schizophrenia for a long time. As HERVs, especially subtype W, are often found to be differentially expressed in the brain of schizophrenic patients [7], this brings up the possibility that they

\begin{tabular}{|c|c|c|c|c|c|}
\hline Gene list & $\mathrm{chr}$ & Itrstart & Itrstop & e-value & Score \\
\hline GABBR1 & chr6 & 29526020 & 29526766 & 0.0 & 1042 \\
\hline MAPK10 & chr4 & 86878290 & 86877559 & 0.0 & 834 \\
\hline MAPK10 & chr4 & 86878341 & 86877841 & 1,00E-160 & 630 \\
\hline DLD & chr7 & 107977734 & 107978228 & 0.0 & 706 \\
\hline NRCAM & chr7 & 107977734 & 107978228 & 0.0 & 706 \\
\hline NR3C2 & chr4 & 148920214 & 148920590 & $1,00 \mathrm{E}-115$ & 464 \\
\hline ATM & chr11 & 107851843 & 107852136 & 2,00E-106 & 430 \\
\hline SLC18A1 & chr8 & 20016820 & 20017144 & $5,00 \mathrm{E}-83$ & 344 \\
\hline SLC18A1 & chr8 & 20017040 & 20017144 & $7,00 \mathrm{E}-12$ & 82 \\
\hline FAAH & chr1 & 46856820 & 46856990 & $2,00 E-44$ & 202 \\
\hline CDC25A & chr3 & 48377262 & 48377349 & $1,00 \mathrm{E}-27$ & 140 \\
\hline MAP4 & chr3 & 48377349 & 48377262 & $1,00 \mathrm{E}-27$ & 140 \\
\hline SHISA5 & chr3 & 48377262 & 48377349 & $1,00 \mathrm{E}-27$ & 140 \\
\hline MAP2 & chr2 & 210171660 & 210171694 & $2,00 \mathrm{E}-05$ & 58 \\
\hline ACTB & chr7 & 5832898 & 5832925 & 0.003 & 50 \\
\hline
\end{tabular}

The regulatory regions were taken from Thurman et al. [16]. The LTR sequence ranges (Itrstart, Itrstop), evalues and scores were calculated using the Blastn program [18]. A large fraction of the regulatory regions identified by Thurman et al. [16] were associated with more than one gene and most genes were also associated with more than one regulatory region. could trigger the downregulation of GABBR1 via its LTR enhancer/regulator.

A full search with the HERV-W LTR as query identified 12 other genes with partial LTR matches overlapping enhancer/regulatory sequences identified by [16], listed in Table 1. Besides GABBR1, which had the strongest match with the LTR sequence of HERV-W, several other schizophrenia-related genes had a partial match. Of those, NRCAM, the neural cell adhesion molecule gene has a well-established association with schizophrenia. Barbeau et al. found that its embryonic isoform was severely decreased in the brain of schizophrenic patients [19].

Interpretation. Long terminal repeats (LTRs) of endogenous viruses have long been known to act as enhancers/ silencers/regulators of gene expression. However, a lack of comprehensive knowledge of regulatory regions associated with their corresponding gene have been lacking till now. As enhancers can be located hundreds of kilobases away from their target gene, a purely computational approach could not possibly identify such regions. The recent study by Thurman et al. [16] made it possible for the first time to analyze the regulatory regions by querying their sequences with functional elements with known (or suspected) pathogenic functionality.

What is the significance of the HERV-W sequences present in the regulatory regions of genes implicated in schizophrenia? Our hypothesis is that their overexpression at the onset of the disease (triggered by an external factor such as an exogenous virus or stress, etc.) leads to their silencing (or downregulation) by hypermethylation. Transposable element silencing as a consequence of DNA methylation is a well-known phenomenon in mammalian species as it is considered the defense system of the host against its "intragenomic parasites", as Yoder et al. calls these elements [20]. In this model silencing genes with a HERV in their regulatory region might be the collateral damage, so to speak, brought upon by the host in its effort to repress the transcription of the HERV-W endogenous retroviruses, ultimately leading to schizophrenia.

Our finding of a full-length HERV-W LTR in the regulatory region of the $\mathrm{GABA}$ receptor $\mathrm{B} 1$ gene GABBR1 implicates it in a subset of cases of schizophrenia. This is in accord with several lines of experimental evidence, notably:

(i) The gene is in the 6p22 genomic region that has been implicated most often in schizophrenia in linkage disequlibrium studies [4,13].

(ii) Postmortem studies suggested that schizophrenia is associated with deficits of GABA-mediated synaptic transmission [21].

(iii) GABBR1 was found to be downregulated in several postmortem studies of schizophrenic patients [5,12] and also in autistic brains [22]. 
Furthermore, our hypothesis of HERV-containing genes silenced by hypermethylation is also supported by experimental evidence, such as:

(iv) HERV-W RNAs tend to be expressed in the cerebrospinal fluid of recent-onset schizophrenic but not in chronic patients [7].

(v) Besides the decreased expression of GABBR1 and that of the neuron adhesion molecule gene (NRCAM), three other genes in Table 1 with the highest scores were also found to be downregulated: the dihydrolipoamide dehydrogenase (DLD) [23] and the mineralocorticoid receptor (NR3C2) [24] in the prefrontal cortex of schizophrenic patients, whereas the mitogen-activated protein kinase 10 (MAPK10) was found to be silenced in several types of cancer [25].

The significance of evidence (iv) is that the lack of HERVs in chronic patients [7] might be due to silencing by hypermethylation, activated by the overexpression of HERV-W RNA at the onset of the disease. It is interesting to note here that Karlsson et al. found recently an inverse correlation between the levels of transcription of HERV-W LTR intergenic elements and their downstream genes [9].

Our model is also supported by the recently observed overexpression of DNA-methyltransferase 1 (DNMT1) in GABAergic interneurons of schizophrenic brains, apparently actively hypermethylating genes with roles in schizophrenia [26]. Interestingly, one of the genes hypermethylated by DNMT1 is reelin [27], which also contains a HERV-W-like sequence (although not an LTR) in its regulatory region (data not shown).

\section{Conclusion}

Taken together, we provided a new hypothesis for the cause of schizophrenia, surmising that the downregulation of several genes containing a HERV-W LTR in their regulatory region might be brought upon by the host in its effort to suppress the expression of the HERV-W sequences, observed usually only at the onset of the disease. It must be noted that our model can be applied to other types of HERVs and other diseases as well. Notably, we also found that one of the genes in our list, MAPK10 is silenced in several types of cancer. Further studies of gene expression and genome-wide methylation might provide a confirmation (or refutal) of our hypothesis.

\footnotetext{
Abbreviations

HERV: Human Endogenous Retrovirus; LTR: Long Terminal Repeat; DHS: DNase I hypersensitive site; GABA: Gamma-Aminobutyric Acid.
}

\section{Competing interests}

The author declares that she has no competing interests.

\section{Reviewers' comments}

Review by Sandor Pongor

The author presents a new hypothesis about the role a GABA receptor, GABBR1 might play in the aetiology of schizophrenia. She bases her theory on a new finding that identifies a HERV-W long terminal repeat in the regulatory region of GABBR1. This is an interesting new theory that would help explain how the ubiquitously present HERV-W elements in recent-onset schizophrenics might contribute to the disease via a full-length LTR regulating the expression of a GABA receptor gene. While, if true, the full picture is probably more complex than stated here (the author herself cites the staggering number of more than 1500 genes being involved in schizophrenia), it is an intriguing theory that might be worthwhile to pursue further, in the light of the fact that the aetiology of the disease is still not known. The author may want to include a few ideas regarding how the hypothesis can be tested.

Quality of written English: Acceptable

I thank Prof. Pongor for his favorable review and suggestions. I somewhat toned down the language of the paper and geared it towards a multi-genic model. I included a sentence at the end regarding experiments to test my hypothesis. It is not easy, of course, as animal models are not very accurate, but the recent advances in genome-wide methylation studies could provide an answer.

Review by Martijn Huynen

The manuscript by Hedi Hegyi argues that it explains the how HERV-W elements play a role in Schizophrenia, based on a combination of observations from the literature about links between Schizophrenia, the expression of HERVW retroviruses after the onset of Schizophrenia, differential expression of GABA receptors in Schizophrenia, the genomic region that has classically been associated with Schizophrenia (6p22.1) and the author's own analysis of the genome and the distribution of published DNAse I accessible sites over HERVW LTRs in Schizophrenia associated genes.

My main criticism is that most of the observations that the hypothesis is based on are themselves hypothesis or associations rather than established causal mechanisms. This makes choosing which evidence is relevant and which is not hard to evaluate. In the genomics area there are so many data sets available that one can be rather eclectic in deciding which ones to choose. I am not accusing the author of doing that, but it is not clear to me why which datasets were chosen. I think it is e.g. pointless to take as input a list of 1600 genes potentially related to schizophrenia.

I thank Martijn Huynen for his thorough review and critical comments, I am going to address them one by one.

I took out the considerations about the 1600 genes and the number of protein binding partners as not very relevant to the main findings.

To my understanding, no causal relationships have been established between GABA receptor expression and schizophrenia or between HERV-W retroviruses and schizophrenia, or between any gene in 6p22.1 and schizophrenia.

No causal relationship has been established between schizophrenia and anything yet. That is the point of my paper, to try to establish a causal relationship between the HERVs and schizophrenia. I hope I made my case stronger in this revised version.

At least one of these relationships is not even consistent: one study shows an increased number of GABA receptors in the brain of schizophrenic patients, while another shows a downregulation of the expression of the gene.

Good point. Digging up the evidence about the increased number of GABA receptors made it clear that it was about the GABA A receptors, therefore I removed it as immaterial to my arguments.

The author's hypothesis, compelling as it may be, therewith appears thus to be build on quicksand. It is not the first time that an analysis of genomics 
data is based on quicksand, but when so many datasets are combined one would like to see some solid mechanisms. Regarding mechanism: as I understand from the literature, expression of HERV-W and other HERV elements after the onset of Schizophrenia has been repeatedly observed. A direct role of human endogeneous retroviruses in Schizophrenia has not been established, so it is not clear to me which role needs to be explained.

It has not been established because nobody gave a satisfactory explanation yet. That does not mean there is no causal relationship!

All in all I do find the conclusions of the manuscript not very tangible and convincing. Lemma $\vee$ of the discussion contains three "mights". To me that is two too many for a convincing hypothesis.

I took out Lemma $V$ as it contained the uncertainty about the increase (GABA A) as opposed to the decrease (GABA B) in the receptors as I explained above. I think (and I hope you agree) my paper and arguments are much more coherent in this revised version, as I was rethinking them and the paper evolved considerably because of that.

\section{Question:}

The Thurman study measured DNAse I accessibility in 125 diverse cell and tissue types. Which of these were chosen for the analysis in Table 1?

The Thurman study did not provide information about tissue types for this particular dataset.

Editorial:

Why was only the HERV-W LTR chosen and not the whole genome, because of their role in regulating gene expression? Could the author mention this earlier in the manuscript and provide a solid reference?

Yes. I inserted now an appropriate reference.

synapse, not "synapsis".

corrected.

Quality of written English: Needs some language corrections before being published.

\section{Acknowledgement}

This research was supported by a Marie Curie reintegration grant IRG-046572 from the European Commission and the grant PD76286 from the Hungarian Scientific Research Fund (OTKA). It is currently supported by the project "CEITEC - Central European Institute of Technology" (CZ.1.05/1.1.00/02.0068) from the European Regional Development Fund.

Received: 10 December 2012 Accepted: 4 February 2013 Published: 7 February 2013

\section{References}

1. Tandon R, Keshavan MS, Nasrallah HA: Schizophrenia, "just the facts" what we know in 2008. 2. Epidemiology and etiology. Schizophr Res 2008, 102(1-3):1-18.

2. Safran M, et al: GeneCards Version 3: the human gene integrator. Database (Oxford) 2010, 2010:baq020.

3. Le-Niculescu H, et al: Towards understanding the schizophrenia code: an expanded convergent functional genomics approach. Am J Med Genet B Neuropsychiatr Genet 2007, 144B(2):129-158.

4. Shi J, et al: Common variants on chromosome 6 p22.1 are associated with schizophrenia. Nature 2009, 460(7256):753-757.

5. Mirnics K, et al: Molecular characterization of schizophrenia viewed by microarray analysis of gene expression in prefrontal cortex. Neuron 2000, 28(1):53-67.

6. Owen MJ, O'Donovan MC, Harrison PJ: Schizophrenia: a genetic disorder of the synapse? BMJ 2005, 330(7484):158-159.

7. Karlsson $\mathrm{H}$, et al: Retroviral RNA identified in the cerebrospinal fluids and brains of individuals with schizophrenia. Proc Natl Acad Sci U S A 2001, 98(8):4634-4639.
8. Perron $\mathrm{H}$, et al: Endogenous retrovirus type W GAG and envelope protein antigenemia in serum of schizophrenic patients. Biol Psychiatry 2008, 64(12):1019-1023.

9. Li F, et al: A systematic evaluation of expression of HERV-W elements; influence of genomic context, viral structure and orientation. BMC Genomics 2011, 12:22.

10. Brudek T, et al: B cells and monocytes from patients with active multiple sclerosis exhibit increased surface expression of both HERV-H Env and HERV-W Env, accompanied by increased seroreactivity. Retrovirology 2009, 6:104.

11. Mudge J, et al: Genomic convergence analysis of schizophrenia: mRNA sequencing reveals altered synaptic vesicular transport in post-mortem cerebellum. PLoS One 2008, 3(11):e3625.

12. Fatemi SH, Folsom TD, Thuras PD: Deficits in GABA(B) receptor system in schizophrenia and mood disorders: a postmortem study. Schizophr Res 2011, 128(1-3):37-43.

13. Arolt $\mathrm{V}$, et al: Eye tracking dysfunction is a putative phenotypic susceptibility marker of schizophrenia and maps to a locus on chromosome $6 p$ in families with multiple occurrence of the disease. Am J Med Genet 1996, 67(6):564-579.

14. Straub RE, et al: Genetic variation in the 6 p22.3 gene DTNBP1, the human ortholog of the mouse dysbindin gene, is associated with schizophrenia. Am J Hum Genet 2002, 71(2):337-348.

15. Capriotti $\mathrm{E}$, et al: Bioinformatics for personal genome interpretation. Brief Bioinform 2012, 13(4):495-512.

16. Thurman RE, et al: The accessible chromatin landscape of the human genome. Nature 2012, 489(7414):75-82.

17. Griffiths DJ: Endogenous retroviruses in the human genome sequence. Genome Biol 2001, 2(6):REVIEWS1017.

18. Morgulis A, et al: Database indexing for production MegaBLAST searches. Bioinformatics 2008, 24(16): 1757-1764.

19. Barbeau D, et al: Decreased expression of the embryonic form of the neural cell adhesion molecule in schizophrenic brains. Proc Natl Acad Sci US A 1995, 92(7):2785-2789.

20. Yoder JA, Walsh CP, Bestor TH: Cytosine methylation and the ecology of intragenomic parasites. Trends Genet 1997, 13(8):335-340.

21. Gonzalez-Burgos G, Fish KN, Lewis DA: GABA neuron alterations, cortical circuit dysfunction and cognitive deficits in schizophrenia. Neural Plast 2011, 2011:723184.

22. Fatemi $\mathrm{SH}$, et al: Expression of $\mathrm{GABA}(\mathrm{B})$ receptors is altered in brains of subjects with autism. Cerebellum 2009, 8(1):64-69.

23. Middleton FA, et al: Gene expression profiling reveals alterations of specific metabolic pathways in schizophrenia. J Neurosci 2002, 22(7):2718-2729.

24. Xing GQ, et al: Decreased expression of mineralocorticoid receptor mRNA in the prefrontal cortex in schizophrenia and bipolar disorder. Int $J$ Neuropsychopharmacol 2004, 7(2):143-153.

25. Ying J, et al: Epigenetic disruption of two proapoptotic genes MAPK10/ JNK3 and PTPN13/FAP-1 in multiple lymphomas and carcinomas through hypermethylation of a common bidirectional promoter. Leukemia 2006, 20(6):1173-1175.

26. Veldic $M$, et al: DNA-methyltransferase $1 \mathrm{mRNA}$ is selectively overexpressed in telencephalic GABAergic interneurons of schizophrenia brains. Proc Natl Acad Sci U S A 2004, 101(1):348-353.

27. Grayson DR, et al: Reelin promoter hypermethylation in schizophrenia. Proc Natl Acad Sci U S A 2005, 102(26):9341-9346.

doi:10.1186/1745-6150-8-5

Cite this article as: Hegyi: GABBR1 has a HERV-W LTR in its regulatory region - a possible implication for schizophrenia. Biology Direct 2013 8:5. 